\title{
Communications
}

\section{Attestation de formation complémentaire}

Examen pour l'obtention de l'attestation de formation complémentaire en phlébologie

Partie écrite (Part 1)

Date: Vendredi, 30 octobre 2015

Lieu: Berne, lors du $16^{e}$ Congrès de l'Union des Sociétés Suisses des Maladies Vasculaires

Délai d'inscription: 20 septembre 2015

Inscription: Dr Jürg Traber, Venenklinik Kreuzlingen, Brückenstrasse 9, 8280 Kreuzlingen,

tél. 07167822 66, fax 07167823 81, e-mail: j.traber[at]venenklinik.ch

Informations: voir sous www.phlebology.ch, lien Certificat de compétence

\section{Société Suisse de Neurologie}

Prix de la Fondation Mogens et Wilhelm Ellermann

En septembre 2015, la Société Suisse de Neurologie attribuera pour la treizième fois le prix de la Fondation Mogens et Wilhelm Ellermann, d'un montant de 20000 francs qui est destiné à récompenser

- un travail scientifique dans le domaine neurologique, qui a été publié lors des deux dernières années ou qui a été accepté pour publication par une revue reconnue sur le plan international,

- une monographie qui n’a pas les caractéristiques d'un traité ni celles d'une revue de la littérature, ou encore

- l'ensemble d'une œuvre scientifique dans le domaine des sciences neurologiques.

Ce travail doit avoir été rédigé par un ou plusieurs chercheurs suisses ou par des chercheurs étrangers en activité dans une clinique ou un institut helvétique. La préférence sera donnée à des auteurs jeunes qui ne sont pas agrégés ou ne le sont que depuis peu.

Les lettres de candidature accompagnées d'un CV et d'une liste des publications (seulement travaux qui sont peer-reviewed) en 5 exemplaires y compris les articles doivent être adressées jusqu'au 31 octobre 2015 au Prof. Dr med. C. L. Bassetti, Chef de service, Clinique et Policlinique neurologique, Hôpital Universitaire de Berne, Inselspital, 3010 Berne.

Terre des hommes

Santé materno-infantile dans les crises humanitaires - offre d'une formation certifiée

La santé materno-infantile recouvre la santé des femmes enceintes, des femmes qui accouchent, des nouveau-nés et des petits enfants de moins de cinq ans. Elle est par essence fragile et davantage encore lors de crises humanitaires. Dans de tels contextes, plus des deux tiers des victimes nécessitant des soins et des mesures de protection appartiennent à ces groupes vulnérables.

Afin de développer les compétences du personnel de pays dits développés en santé materno-infantile dans des contextes d'urgence humanitaire, la Haute Ecole de Santé Vaud (HESAV) et la Fondation Terre des

\section{Sujets actuels de forum}

Joignez la discussion en ligne sur www.saez.ch
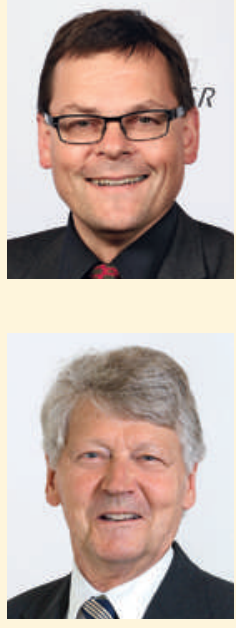

Dr med. Tarzis Jung

Président de la Société Suisse de Radiologie (SGR-SSR)

Médecin-chef de I'Institut de Radiologie et de Médecine nucléaire, Stadtspital Waid, Zurich

«Non, une IRM n'est pas toujours nécessaire.»

Réponse à la contribution sur les examens spéciaux

Dr med. Max Giger, président de FMH Services, conseiller scientifique de Dialog Ethik, Winterthour

\section{Serment des médecins}

Nous avons besoin d'un nouveau serment obligatoire pour les médecins 
hommes (Tdh), à Lausanne, lancent en 2016 un certificat de formation continue (Certificate of Advanced Studies [CAS]) en cours d'emploi.

L'accès à l'eau, à la nourriture (y compris à l'allaitement maternel), aux soins médicaux, à un abri devient problématique lors de catastrophes humanitaires et ceci avec des effets dévastateurs sur la santé des petits enfants: malnutrition aiguë, diarrhée, infection respiratoire, paludisme. Les nouveau-nés sont particulièrement vulnérables avec une nette augmentation de leur mortalité. La santé des femmes enceintes et des femmes qui accouchent est aussi fortement péjorée. En plus des lourdes menaces sur leur santé, les victimes survivantes sont souvent à haut risque de viol, enlèvement et exploitation.

Aujourd'hui, les crises humanitaires à travers le monde sont multiples et, malheureusement, rien ne laisse supposer qu'il en sera différent demain. Quatre-vingt millions de personnes ont besoin d'assistance humanitaire. Les trois quarts d'entre elles sont des femmes et des enfants.
Sauver le plus grand nombre possible de vies de mères et d'enfants avec des moyens cliniques et paracliniques limités, soutenir les autorités sanitaires locales, faciliter la réhabilitation des centres de santé communautaire: tels sont les trois grands principes qui régissent la formation proposée par HESAV et Tdh.

$\mathrm{Au}$ terme de leur cursus, les étudiant-e-s auront acquis les connaissances nécessaires pour identifier les besoins en santé maternoinfantile et mettre en œuvre des interventions appropriées, efficaces et efficientes destinées aux mères, aux nouveau-nés et aux jeunes enfants en situation de crises humanitaires complexes.

Le CAS débute en janvier 2016 et se termine en novembre. Il comprend 120 heures d'enseignement frontal (dix-sept jours répartis sur un an), à Lausanne. En plus, quelque 200 heures de travail à domicile sont à prévoir. En fin de cours, un examen est prévu. Sa réussite donne droit à 10 crédits européens ECTS et à 37 crédits de la Société Suisse de Pédiatrie. D’autres crédits FMH sont en cours de demande.
A qui s'adresse ce CAS? Aux médecins, aux infirmier-ère-s et aux sages-femmes diplômés provenant des pays dits développés et prévoyant d'intervenir, ou intervenant déjà, dans le domaine de la santé maternoinfantile en situation humanitaire. Le délai d'inscription court jusqu'à fin novembre 2015. Son coût est de 4300 CHF. Sous certaines conditions des bourses d'étude sont à disposition.

Pour plus d'informations générales: www. hesav.ch/postgrade/formation/cas-hesav/ cas-en-sant\%C3\%A9-materno-infantile-dansles-crises-humaintaires

Pour davantage de renseignements personnalisés: michel.roulet[at]tdh.ch 